OPEN ACCESS

Edited by:

Edmund C. Lalor,

University of Rochester,

United States

Reviewed by:

Rifai Chai,

University of Technology Sydney,

Australia

Gerard Martin Loughnane,

Trinity College Institute of

Neuroscience, Ireland

*Correspondence:

Christian C. Joyal

christian.joyal@uqtr.ca

Received: 07 January 2018 Accepted: 13 June 2018

Published: 09 July 2018

Citation:

Joyal CC, Neveu S-M, Boukhalfi T, Jackson PL and Renaud P (2018) Suppression of Sensorimotor Alpha Power Associated With Pain

Expressed by an Avatar: A

Preliminary EEG Study.

Front. Hum. Neurosci. 12:273. doi: 10.3389/fnhum.2018.00273

\section{Suppression of Sensorimotor Alpha Power Associated With Pain Expressed by an Avatar: A Preliminary EEG Study}

\author{
Christian C. Joyal ${ }^{1,2 *}$, Sarah-Michelle Neveu ${ }^{1}$, Tarik Boukhalfi ${ }^{1}$, Philip L. Jackson ${ }^{3}$ \\ and Patrice Renaud ${ }^{1,4}$
}

'Laboratory of Virtual Reality Applications in Psychiatry (ARVIPL), Research Center, Philippe-Pinel Institute of Montreal, Montreal, QC, Canada, ${ }^{2}$ Cognition, Neuroscience, Affect and Behavior Research Group (CogNAC), Psychology Department, University of Quebec at Trois-Rivières, Trois-Rivières, QC, Canada, ${ }^{3}$ Psychology Department, University Mental Health Institute of Quebec (CRIUSMQ) and Laval University, Quebec, QC, Canada, ${ }^{4}$ Psychology Department, University of Quebec in Outaouais, Gatineau, QC, Canada

Several studies using functional magnetic resonance imaging (fMRI) showed that empathic capabilities are associated with the activation (and deactivation) of relatively specific neural circuits. A growing number of electroencephalography studies also suggest that it might be useful to assess empathy. The main goal of this study was to use quantitative electroencephalography (qEEG) to test whether observation of pain expressed by an avatar (virtual reality) induces a suppression of alpha waves over sensorimotor cortical areas, as it is observed with human stimuli. Not only was it the case, but also the magnitude of alpha suppression was correlated with perspectivetaking capacity of participants. Both empathy levels and magnitude of sensorimotor alpha suppression (SAS) were significantly higher in women than men. Interestingly, a significant interaction emerged between levels of individual empathy and specificity of experimental instructions, where SAS in participants with good perspective-taking was higher during passive observation of the distressed avatar, while the opposite was true during an active (trying to understand) condition. These results suggest that: (1) synthetic characters are able to elicit SAS; (2) SAS is indeed associated with perspective-taking capacities; (3) Persons with poorer perspective-taking capacities can show significant SAS when proper instructions are provided. Therefore, qEEG represents a low-cost objective approach to measure perspective-taking abilities.

Keywords: empathy, assessment, electroencephalography, qEEG, perspective taking, alpha suppression, avatar

\section{INTRODUCTION}

Currently, in clinical settings, empathy is usually assessed through self-report questionnaires (i.e., the Interpersonal Reactivity Index, Davis, 1983), which are highly susceptible to bias due to false responses, social desirability, and other factors (e.g., Van de Mortel, 2008). Identifying a neurobiological correlate of empathy would make it possible to develop an objective assessment. Several experimental studies have demonstrated that functional magnetic resonance imaging (fMRI) allows the visualization and quantification of key cortical and subcortical brain activation 
associated with empathy (e.g., Jackson et al., 2005; see Bernhardt and Singer, 2012; for a review). fMRI, however, is costly and not accessible in most clinical or forensic settings. The main goal of the present study was to confirm the capacity of a lowcost, available, portable neuroimaging technique-quantitative electroencephalography ( $\mathrm{qEEG}$ ) - to evaluate empathy levels. EEG records local electric potentials generated by the cerebral cortex and qEEG mathematically breaks down the frequency spectrum of electrical waves. This approach allows measurement of regional (different cortical areas) power (or amplitude) of particular oscillatory bands with high precision for both frequency (e.g., 1-Hz gradient) and time (1-s gradient). Given that empathic traits and states are associated with regional cortical modulation, qEEG might be useful to evaluate an individual's empathy levels.

One of the most exciting discoveries in neuroscience over the past half-century is that of mirror neurons, i.e., frontoparietal cortical cells that are activated (mirroring) in human and non-human primates during observation of a congener performing an act or behavior (Rizzolatti and Craighero, 2004). Mirror neuron responses activate the primary motor cortex (Hari et al., 1998; Muthukumaraswamy et al., 2004), forming a neural system that is hypothesized to promote prosocial behaviors through linking perception (seeing and understanding someone in distress) to action (providing assistance; Preston and de Waal, 2002; Pineda, 2005). Given that similar reactive activations are also observed in the somatosensory cortex (the other side of the central fissure) when a person observes painful stimuli or another person in pain (e.g., Avenanti et al., 2005; see Keysers and Gazzola, 2010; for a review), both primary motor and somatosensory cortices represent an important part of the cortical areas involved in the neural foundations of social interactions (Perry et al., 2011; Vanderwert et al., 2013) and empathy (Bernhardt and Singer, 2012).

qEEG is perfectly suited to measure sensorimotor cortical activation. First, sensorimotor cortex correspond to middle electrodes (T7-C3-Cz-C4-T8), which are less sensitive to eye blinks than anterior electrodes. Second, regional cortical asynchrony induces a power reduction in alpha wave frequencies (8-13 Hz), especially those surrounding 8-10 Hz (Pfurtscheller and Lopes da Silva, 1999; Goldman et al., 2002), which is easily measured with Fast Fourier Transformation (FFT) of the EEG signal. The sensorimotor alpha suppression (SAS) is thought to reflect a downstream cortical activation generated by the mirror neurons (Pineda, 2005). Interestingly, a growing number of qEEG studies suggest that empathic capacities are also associated with the magnitude of SAS (Cheng et al., 2008a; Yang et al., 2009; Perry et al., 2010; Woodruff et al., 2011; Moore et al., 2012; Hoenen et al., 2013, 2015; see also Babiloni et al., 2012; for more anterior regions of interest; Ono et al., 2009; for using the corollary enhancement of beta power in central locations).

Both qEEG source localization (Yang et al., 2009; Moore et al., 2012), and magneto-encephalography source modeling (Whitmarsh et al., 2011) have confirmed that SAS associated with empathic response is highest above the sensorimotor cortical cortex, more particularly on the posterior bank of the central sulcus (Cheng et al., 2008b). Interestingly, SAS may be significantly stronger in women than in men (Cheng et al., 2006, 2008a; Yang et al., 2009; see also Han et al., 2008; Schulte-Rüther et al., 2008), which would be compatible with the well-established fact that women, in general, possess higher empathy capacities and social sensitivity than men (e.g., Baron-Cohen et al., 2005; Toussaint and Webb, 2005). Gender difference in SAS is not systematically reported, however (Perry et al., 2011), and deserves further investigation.

SAS induced by a painful expression exhibited by another person is viewed as a gating mechanism that disinhibits sensory cortices, helping to understand the pain of others (Whitmarsh et al., 2011; Moore et al., 2012). Peng et al. (2015) suggest that central (or sensorimotor) alpha oscillatory modulation associated with pain perception is determined by sensoridiscriminative, affective motivational and cognitive-modulative aspects of pain experience. Therefore, qEEG represents a promising, accessible, and affordable technique for evaluating empathy and its sub-components that avoids any self-report bias.

qEEG might also help enhance empathy through neurofeedback. Neurofeedback is an operant learning technique based on brain-computer interfaces. Although evidence-based data with qEEG neurofeedback generally concerns attention deficits and impulsivity (Arns et al., 2014), it has been used successfully to train other capacities, both for clinical (Simkin et al., 2014) and non-clinical (Gruzelier, 2014) purposes. Among these trainable capacities is empathy (Cavazza et al., 2014; Moll et al., 2014; Yao et al., 2016).

However, many questions need to be addressed before SAS can be used as a correlate of empathy, let alone a neurofeedback target. First, some studies (but not all, e.g., Perry et al., 2011; Woodruff et al., 2011; Hoenen et al., 2013) do not demonstrate its specificity as they fail to provide comparisons with alpha suppression that occurs simultaneously in other (non-central) cortical regions. The occipital cortices, most notably, should be considered because it is well known that alpha power is suppressed posteriorly by visual stimulation or increased attention load (Thut et al., 2006).

Second, the majority of existing studies elicited SAS through presentation of simple motor stimuli (e.g., observing or performing movements; Perry et al., 2011; Woodruff et al., 2011). Only a handful of qEEG investigations used presentation of stimuli related to empathy (e.g., signs of distress or pain), and these stimuli were typically limited to body parts, usually the hands (e.g., hands being pricked, cut, or crushed; Yang et al., 2009; Perry et al., 2010). One exception used video clips of human actors in painful (sad) situations (Hoenen et al., 2013), although the mode of expression was verbal (story telling), which is not well suited for neurofeedback training. Past studies also used brief stimulus presentations, commonly ranging from $1.7 \mathrm{~s}$ to $5 \mathrm{~s}$ for pictures (e.g., Yang et al., 2009; Perry et al., 2010; Moore et al., 2012; Hoenen et al., 2015), up to $80 \mathrm{~s}$ for video clips (Cheng et al., 2008a; Woodruff et al., 2011; Hoenen et al., 2013). It remains to be seen whether SAS can also be detected with longer (and noisier) stimulus presentations (e.g., 120 s), which is mandatory for neurofeedback training (Budzynski et al., 2009).

Third, although visual stimulation and virtual reality represent the best options for conducting neurofeedback studies, 
very few EEG studies used virtual agents expressing pain to generate brain response associated with empathy (Cavazza et al., 2014). While it is known that avatars can elicit empathic responses in humans (e.g., Paiva et al., 2005; Cheetham et al., 2009; Joyal et al., 2014), their capacity to elicit SAS associated with empathy remains to be demonstrated (de Borst and de Gelder, 2015). This point is crucial to the eventual development of neurofeedback training, which could involve, for instance, presenting avatars expressing different types and intensities of emotions in response to brain wave characteristics of the participant.

Finally, empathy is a multifaceted construct and its sub-components should be considered separately in EEG studies. The four-factor model of empathy proposed by Davis (1983) is still the best validated model to date (Chrysikou and Thompson, 2016). According to that model, empathy encompasses four different capacities: (1) perspective taking, or the tendency to spontaneously adopt the psychological point of view of others; (2) cognitive fantasizing, or the tendency to transpose ourselves imaginatively into fictional situations; (3) empathic concern, or the tendency to have feelings of sympathy or compassion oriented toward others; and (4) personal distress, or the tendency to have self-oriented feelings of personal anxiety or fear in response to seeing others in distress (Davis, 1983; Chrysikou and Thompson, 2016). To be clinically relevant, a biological correlate of empathy should be sensitive to perspective taking, as a defect in this capacity is a classic predictor of low communication skills, antisocial behaviors, and interpersonal violence (Chandler, 1973). To date, two studies have reported that perspective-taking capacities have a modulating effect on SAS (Perry et al., 2010; Hoenen et al., 2013), and a third reported a specific and significant correlation between SAS and perspective-taking capacities, although the study was based on motor (moving fingers) stimuli (Woodruff et al., 2011). It remains to be seen if the same association would be observed within an empathic context, especially if the character presented is synthetic.

The main goal of this preliminary investigation was to assess the capacity of avatars to elicit empathy-related SAS, while considering extraneous factors such as non-central alpha suppression and presentation of equivalent stimuli not expressing pain. Another objective of this study was to measure the strength of association between SAS and individual levels of empathy and its sub-components. We attempted to confirm the superiority of women vs. men in empathy-related SAS. Finally, this study aimed at providing some guidelines for future neurofeedback protocols. The following hypotheses were tested: H1: the magnitude of alpha wave suppression elicited by observation of expressions of pain will be significantly higher in central (sensorimotor) than occipital electrodes; H2: a dynamic avatar expressing pain will elicit SAS with a magnitude significantly higher than that evoked by the same avatar not expressing pain; H3: the magnitude of SAS elicited by observation of expressed pain will be significantly associated with the empathy capacities of participants; H4: women will show, on average, a significantly higher magnitude of SAS during observation of expressed pain than men.

\section{MATERIALS AND METHODS}

\section{Participants}

Based on effect sizes obtained in previous studies (e.g., Hoenen et al., 2013), 24 right-handed adults were recruited from the general population through online classified advertising to participate in this study (mean age: $26.7 \pm 4.6,20-40$ ). The sample consisted equally of men and women $(n=12)$, all screened for histories of neurological or psychiatric conditions through phone pre-screening and an on-site questionnaire. Participants had to refrain from drinking alcohol for $24 \mathrm{~h}$ prior to testing, and from drinking coffee (or any beverage containing caffeine) and smoking tobacco for $12 \mathrm{~h}$ prior to testing.

\section{Questionnaires}

Levels of empathy were assessed individually with a validated French version (Gilet et al., 2013) of the International Reactivity Index (IRI), a self-report questionnaire that assesses four components of empathy: perspective taking (the tendency to spontaneously adopt the psychological point of view of others), fantasizing (the tendency to transpose ourselves imaginatively into fictional situations), empathic concern (having feelings of sympathy or compassion toward others), and personal distress (self-oriented feelings of personal anxiety or fear in response to seeing others in distress; Davis, 1983; Chrysikou and Thompson, 2016). In the French version, items are rated on a 7-point scale ranging from 1 (does not describe me well) to 7 (describes me very well), generating higher mean scores than the original 5-point scale (1983).

\section{Neurophysiological Material and Virtual Reality Environment}

A 32-electrode EEG cap (Acticap, extended 10-20 system, Brain Products, LLD) equipped with active wireless electrodes (ActiChamp amplifier paired with a MOVE system, Brain Products) was used to record electrical cortical activity (Figure 1). Participants were seated in a CAVE-like, 4-wall immersive environment (iCube, Viz-Tek Inc.) wearing 3D active shutter glasses (EdgeVR, Volfoni, Inc.) and headphones fitted with the wireless Acticap (Figure 1). This EEG system was chosen because it is particularly robust against electromagnetic fields (active electrodes with a co-integrated amplifier and noise subtraction; Metting Van Rijn et al., 1996; Usakli, 2010).

A 2-min period of habituation was first allowed, in which participants could freely explore the content of a neutral animation presented in $3 \mathrm{D}$. Because simple observation of movement is known to suppress central alpha wave (e.g., Muthukumaraswamy et al., 2004), experimental conditions involved a non-moving avatar, a moving avatar, and the same moving avatar expressing pain. Stereoscopic stimuli were presented for $2 \mathrm{~min}$ each and consisted of the four following items (see Boukhalfi et al., 2015 for technical details): (1) change of the environment background from black to lighter gray (baseline condition, eye-open, luminosity modification); (2) presentation of a whole-body, adult Caucasian male avatar, seated in front of the same gray background, dressed in black and gray, audibly and regularly breathing (neutral condition); 

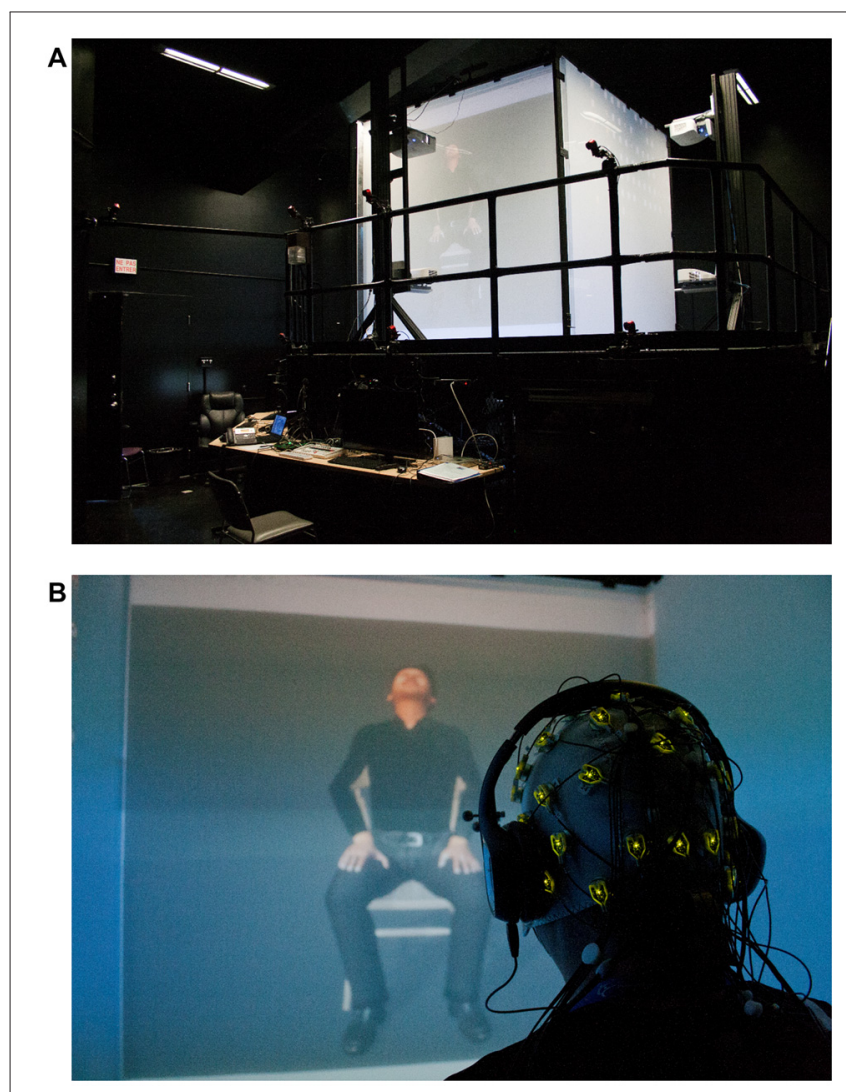

FIGURE 1 | (A) The CAVE-like environment projecting the avatar;

(B) a participant wearing the wireless EEG Acticap, headphones and the 3D goggles observing the avatar expressing pain.

(3) presentation of the same seated avatar with the same audible breathing but moving forward and backward with hands on knees (movement condition); and (4) presentation of the same seated and moving avatar expressing pain facially and orally (pain condition). Facial expressions of emotions by these stimuli had been previously validated with another non-clinical sample of participants (Joyal et al., 2014).

Participants were asked either to simply observe the virtual character as an external object, without trying to understand or feel his emotions (Observation) or to try to understand and feel the emotion expressed by the virtual character (Empathy). Stimuli were presented six times in total-two different instructions (observe or empathize) across four different conditions (baseline, neutral, movement and expressed pain)-with the order of presentation counterbalanced for instruction (observation or empathy) and conditions (Neutral $\rightarrow$ Movement $\rightarrow$ Pain, or Pain $\rightarrow$ Movement $\rightarrow$ Neutral). Each presentation was followed by a 2 -min rest period.

\section{Data Acquisition}

This study is based on continuous qEEG signals recorded at bilateral sensorimotor (T7-C3-Cz-C2-T8) and occipital (O3Oz-O2) sites (Recorder software, Brain Products). The more lateral T7 and T8 electrodes were added to the classical central electrodes $(\mathrm{C} 3-\mathrm{Cz}-\mathrm{C} 4)$ to include lateral parts of the

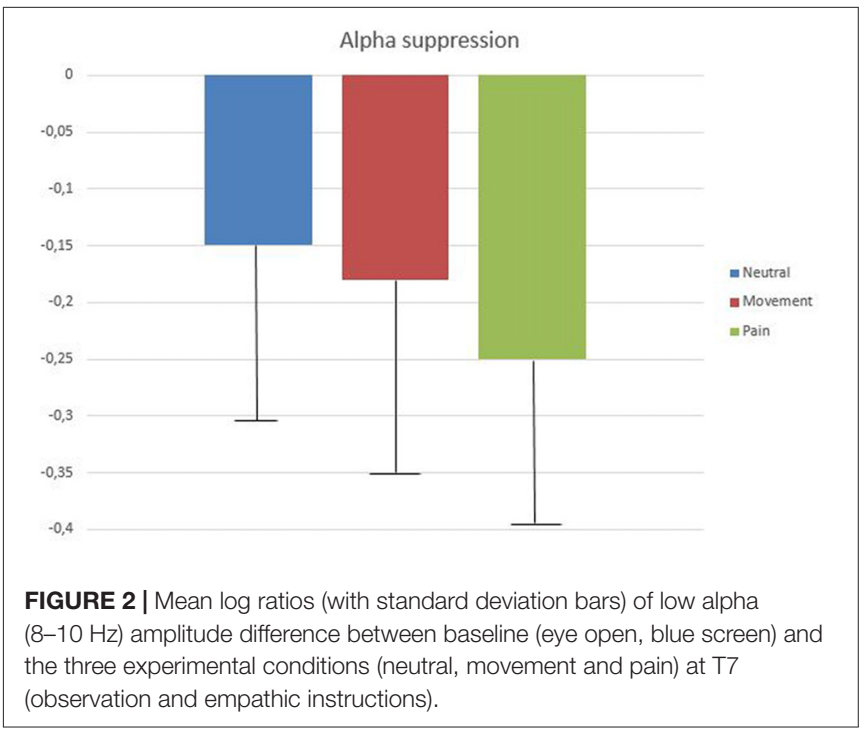

sensorimotor cortex (Fabbri-Destro and Rizzolatti, 2008), usually overlooked by previous EEG studies. Data were referenced at $\mathrm{FPz}$ and re-referenced offline with bilateral mastoids to reduce eye-blink contamination. Electrical impedance was held below $25 \mathrm{k} \Omega$, as required with active EEG electrodes. Data were sampled at $500 \mathrm{~Hz}$ and filtered with bandpass set at $0.01 \mathrm{~Hz}$ (high-pass) and $70 \mathrm{~Hz}$ (low-pass), and a notch filter for the $60 \mathrm{~Hz}$ frequency (North American electrical noise). Signals exceeding $\pm 100 \mu \mathrm{V}$ (muscle artifacts) were automatically rejected.

\section{Data Analyses}

Data processing followed the procedure of previous studies that used SAS to evaluate social cognition and empathy (Perry et al., 2011; Woodruff et al., 2011). First, both sensorimotor and occipital sites were recorded to make it possible to distinguish between alpha modulation generated by observation of expressed pain (sensorimotor) vs. alpha wave reaction to more general modifications of the visual environment (occipital) or attentional processes (widespread). Second, individual EEG outputs were inspected for artifacts by two independent raters (including a certified medical EEG technician) until a $100 \%$ inter-rater agreement was reached. At least $60 \mathrm{~s}$ of artifact-free EEG data was required (and obtained in all cases) to achieve minimal stability for spectral decomposition (qEEG). Third, records were divided into $1 \mathrm{~s}$ segments (minimum of 60 segments), and for each segment the integrated power in the $8-13 \mathrm{~Hz}$ range was computed using a Fast Fourier Transform (FFT) obtained at $1 \mathrm{~Hz}$ intervals with a Hanning window. Fourth, segments were averaged for each condition (baseline, neutral, movement and pain). Given that the absolute signal power is sensitive to individual differences (e.g., skull density, scalp thickness, baldness, electrode impedance), ratios were computed for each condition based on the baseline data (i.e., neutral/baseline; movement/baseline; pain/baseline). Finally, ratios were log transformed because they are not normally distributed (Pineda and Oberman, 2006). A negative log ratio under indicates a suppression of power compared to the baseline. Therefore, the 
dependant variable was a suppression index, calculated as the logarithm of the ratio of the alpha power during each condition relative to the alpha power during the baseline condition (Perry et al., 2011). Given our objective to obtain guidelines for neurofeedback protocols, exploratory analyses were conducted with $8-10 \mathrm{~Hz}$ vs. $8-13 \mathrm{~Hz}$ bands at each electrode (data not shown). In accord with early studies of SAS (Cochin et al., 1998, 1999), empathy-related SAS was optimal in the $8-10 \mathrm{~Hz}$ band. It was also highest, on average, at the T7 electrode (left lateral sensorimotor cortex). Therefore, all remaining analyses are based on $8-10 \mathrm{~Hz}$ suppression at T7.

\section{Statistical Analyses}

An ANCOVA was conducted to control for occipital alpha suppression (covariate) and to test inter-factor interactions: 2 (gender) $\times 2$ (level of dispositional empathy: low vs. high) $\times 2$ (instruction: simply observe vs. empathize) with SAS as the dependant, repeated measure (neutral, movement, pain). Greenhouse-Geisser corrections were applied to the degree of freedom when necessary (i.e., if data sphericity was not attained). Effect sizes (corresponding to Pearson's coefficient $r$ ) of $0.10,0.30$ and 0.50 were considered as small, medium and large, respectively. The strength of association between the magnitude of T7 alpha suppression and that of subcomponents of empathy was also assessed with $r$ (bivariate Pearson comparisons).

\section{Ethical Considerations}

A compensation of $\$ 50$ was given to each participant at the end of the experiment. The study was approved by the ethics committees of the Philippe-Pinel Institute of Montreal and the University du Québec en Outaouais (UQO). This study was carried out in accordance with the recommendations of the Canadian "Tri-Council Policy Statement: Ethical Conduct for Research Involving Humans (TCPS)" with written informed consent from all subjects. All subjects gave written informed consent in accordance with the Declaration of Helsinki.

\section{RESULTS}

The 2 (gender) $\times 2$ (level of empathy) $\times 2$ (instruction) ANCOVA (controlling for alpha modulation at occipital sites) with repeated measures (neutral, movement, and pain) confirmed that the three types of avatar presentation induced significant SAS (compared with the baseline condition), which was significantly greater during the pain condition $(M=-0.25$ $\pm 0.17)$ than during both the neutral $(M=-0.146 \pm 0.22)$ and movement $(M=-0.18 \pm 0.18)$ conditions $\left(F_{(1,39)}=3.83\right.$, $p<0.05 ; r=0.49$; post hoc: pain vs. neutral, $p<0.001$; pain vs. movement, $p=0.003$; neutral vs. movement, $p>0.05$; Figure 2). Main effects for level of empathy (low: $M=-0.216 \pm 0.21$ vs. high: $\left.M=-0.284 \pm 0.25 ; F_{(1,39)}=9.9, p=0.03 ; r=0.44\right)$ and gender (men: $-0.229 \pm 0.24$ vs. women: $-0.272 \pm 0.23$; $\left.F_{(1,39)}=4.6, p<0.05 ; r=0.33\right)$ were also significant, with significantly higher alpha suppression in participants with high empathy scores and in women.
The effect of instructions (observe vs. empathize) was not statistically significant $\left(F_{(1,39)}=0.39, p>0.05 ; r=0.1\right)$. In fact, the simple observation of the avatar tended to induce greater SAS (all types of presentations still taken together), than trying to empathize with it (observe: $M=-0.219 \pm 0.23$ vs. empathize: $\left.M=-0.166 \pm 0.22 ; F_{(1,47)}=2.69, p=0.10, r=0.22\right)$. No interaction was statistically significant.

Limiting the analyses to the pain condition revealed that the magnitude of SAS in both men $(M=-0.377 \pm 0.23)$ and women $(M=-0.404 \pm 0.24)$ with high empathy levels was significantly higher than that for both men $(M=-0.206 \pm 0.18)$ and women $(M=-0.212 \pm 0.21)$ with low empathy levels $\left(F_{(1,23)}=4.34, p=0.05, r=0.42\right)$. However, the interaction between the instruction condition (simply observing distress vs. attempting to empathize with the distressed avatar) and the level of empathy (low vs. high) approached significance $\left(F_{(1,47)}=2.96\right.$, $p=0.09, r=0.27)$, indicating that simple observation tended to be sufficient to elicit significant central alpha suppression in persons with high levels of empathy, whereas attempting empathize with the avatar was necessary (and successful) to elicit alpha suppression in persons with low levels of empathy.

Finally, bivariate two-way Pearson correlations showed that the magnitude of SAS provoked by the distressed avatar was associated (nearly significant) with the perspective-taking capacities of the participants under the observing $(r=0.398$, $p=0.054)$ but not the empathizing conditions. No other correlations approached significance.

\section{DISCUSSION}

The main goal of this study was to determine whether an avatar expressing pain can induce a significant SAS in humans. In accord with fMRI investigations (e.g., Gobbini et al., 2011), this study found that a synthetic agent can activate brain regions associated with empathy. It was found that SAS was evoked by observation of an avatar expressing pain and was significantly stronger than that evoked by the same avatar executing the same movement without expressing pain. Therefore, SAS was not simply due to viewing movements (e.g., Muthukumaraswamy et al., 2004). In addition, SAS did not simply reflect a posterior (occipital) alpha modulation associated with attentional processing (e.g., Thut et al., 2006), because the latter was entered as a co-variable in the analyses. These results represent a partial answer to the questions raised by de Borst and de Gelder (2015), who correctly wondered if using avatars for the neurobiological assessment of empathy is appropriate.

Another goal of this study was to confirm that SAS is associated with individual trait empathy or its sub-components. In accordance with Woodruff et al. (2011), it was found that the magnitude of SAS evoked by the observation of pain correlated with perspective-taking capacities, but not with the three other components. Therefore, the usefulness of SAS to assess empathy seems to concern perspective-taking capacities in particular.

As expected (Cheng et al., 2006, 2008a; Yang et al., 2009), a higher magnitude of SAS was found in women, on average, than 
in men. Also in accord with previous investigation (Moore et al., 2012), instructing participants to simply observe vs. to attempt to understand what the avatar was feeling had no effect, on average, on the magnitude of SAS. Interestingly, however, participants with higher empathy only needed to observe the distressed avatar to show significant SAS, whereas participants with lower empathy capacities needed to consciously try to understand the feelings of the avatar to show a significant SAS. These results suggest not only the presence of an automatic brain mechanism in empathic persons but also that the mechanism can be elicited through conscious effort. This possibility should be tested with clinical populations that lack empathy.

This study should not serve as a blueprint for neurofeedback paradigms, however. Although empathy-related SAS was higher, on average, at T7, it was not necessarily the case for all participants. As shown in the results, standard deviations (interindividual variation) were elevated (much more than variations of empathy levels). Therefore, neurofeedback paradigms aiming at modulating empathy-related SAS will have to target cortical sites determined individually, as they might differ from one person to another.

In conclusion, this study confirms suggestions that qEEG and sensorimotor alpha waves can serve to assess empathy (Yang et al., 2009; Perry et al., 2010; Woodruff et al., 2011; Hoenen et al., 2013), and, more specifically, perspective-taking capacities. The effectiveness of using avatars to evaluate and, eventually, to train cerebral response associated with empathy was also demonstrated. The search for a single brain site to

\section{REFERENCES}

Arns, M., Heinrich, H., and Strehl, U. (2014). Evaluation of neurofeedback in ADHD: the long and winding road. Biol. Psychol. 95, 108-115. doi: 10.1016/j. biopsycho.2013.11.013

Avenanti, A., Bueti, D., Galati, G., and Aglioti, S. M. (2005). Transcranial magnetic stimulation highlights the sensorimotor side of empathy for pain. Nat. Neurosci. 8, 955-960. doi: 10.1038/ nn 1481

Babiloni, C., Buffo, P., Vecchio, F., Marzano, N., Del Percio, C., Spada, D., et al. (2012). Brains "in concert": frontal oscillatory $\alpha$ rhythms and empathy in professional musicians. Neuroimage 60, 105-116. doi: 10.1016/j.neuroimage. 2011.12.008

Baron-Cohen, S., Knickmeyer, R. C., and Belmonte, M. K. (2005). Sex differences in the brain: implications for explaining autism. Science 310, 819-823. doi: 10.1126/science.1115455

Bernhardt, B. C., and Singer, T. (2012). The neural basis of empathy. Annu. Rev. Neurosci. 35, 1-23. doi: 10.1146/annurev-neuro-062111-1 50536

Boukhalfi, T., Joyal, C., Bouchard, S., Neveu, S. M., and Renaud, P. (2015). Tools and techniques for real-time data acquisition and analysis in brain computer interface studies using qEEG and eye tracking in virtual reality environment. IFAC-PapersOnLine 48, 46-51. doi: 10.1016/j.ifacol.2015.06.056

Budzynski, T. H., Budzynski, H. K., Evans, J. R., and Abarbanel, A. (Eds). (2009). Introduction to Quantitative EEG and Neurofeedback: Advanced Theory and Applications. 2nd Edn. New York, NY: Academic Press.

Cavazza, M., Aranyi, G., Charles, F., Porteous, J., Gilroy, S., Klovatch, I., et al. (2014). "Towards empathic neurofeedback for interactive storytelling," in 2014 Workshop on Computational Models of Narrative OpenAccess Series in Informatics (OASIcs), 5th Edn. (Vol. 41) eds M. A. Finlayson, J. C. Meister and E. G. Bruneau (Dagstuhl, Germany: Schloss Dagstuhl-Leibniz-Zentrum fuer Informatik), 42-60. use in such training proved to be futile, however, as intersubject variability was too high. While evaluative qEEG studies for empathy can make use of the average signals of all central electrodes, future neurofeedback protocols will have to determine individually which region is most effective for each participant. Finally, the usefulness of the present results should be confirmed in future qEEG studies that use participants with known empathy deficits recruited from clinical and forensic settings.

\section{AUTHOR CONTRIBUTIONS}

CJ initiated and contributed to experimental design, conducted the literature review, analyzed and interpreted the data and wrote the manuscript. S-MN collected the data and contributed to experimental design and data interpretation. TB created the stimuli, programed the presentation scripts and coordinated the multiplateform interface. PJ contributed to experimental design, data interpretation and revision of the manuscript. PR initiated and contributed to experimental design, revision of the manuscript and data interpretation.

\section{FUNDING}

This study was funded by a research team grant from the Fonds de Recherche Nature et Technologie du Québec (2014-PR-173102).

Chandler, M. J. (1973). Egocentrism and antisocial behavior: the assessment and training of social perspective-taking skills. Dev. Psychol. 9, 326-332. doi: $10.1037 / \mathrm{h} 0034974$

Cheetham, M., Pedroni, A., Antley, A., Slater, M., and Jäncke, L. (2009). Virtual milgram: empathic concern or personal distress? Evidence from functional MRI and dispositional measures. Front. Hum. Neurosci. 3:29. doi: 10.3389/neuro.09.029.2009

Cheng, Y.-W., Lee, P. L., Yang, C. Y., Lin, C. P., Hung, D., and Decety, J. (2008a). Gender differences in the mu rhythm of the human mirror-neuron system. PLoS One 3:e2113. doi: 10.1371/journal.pone.0002113

Cheng, Y.-W., Yang, C. Y., Lin, C. P., Lee, P. L., and Decety, J. (2008b). The perception of pain in others suppresses somatosensory oscillations: a magnetoencephalography study. Neuroimage 40, 1833-1840. doi: 10.1016/j. neuroimage.2008.01.064

Cheng, Y.-W., Tzeng, O. J., Decety, J., Imada, T., and Hsieh, J. C. (2006). Gender differences in the human mirror system: a magnetoencephalography study. Neuroreport 17, 1115-1119. doi: 10.1097/01.wnr.0000223393. 59328.21

Chrysikou, E. G., and Thompson, W. J. (2016). Assessing cognitive and affective empathy through the interpersonal reactivity index: an argument against a two-factor model. Assessment 23, 769-777. doi: 10.1177/1073191115 599055

Cochin, S., Barthelemy, C., Lejeune, B., Roux, S., and Martineau, J. (1998). Perception of motion and qEEG activity in human adults. Electroencephalogr. Clin. Neurophysiol. 107, 287-295. doi: 10.1016/s0013-4694(98)00071-6

Cochin, S., Barthelemy, C., Roux, S., and Martineau, J. (1999). Observation and execution of movement: similarities demonstrated by quantified electroencephalography. Eur. J. Neurosci. 11, 1839-1842. doi: 10.1046/j.14609568.1999.00598.x

Davis, M. H. (1983). Measuring individual differences in empathy: evidence for a multidimensional approach. J. Pers. Soc. Psychol. 44, 113-126. doi: $10.1037 / 0022-3514.44 .1 .113$ 
de Borst, A. W., and de Gelder, B. (2015). Is it the real deal? Perception of virtual characters versus humans: an affective cognitive neuroscience perspective. Front. Psychol. 6:576. doi: 10.3389/fpsyg.2015.00576

Fabbri-Destro, M., and Rizzolatti, G. (2008). Mirror neurons and mirror systems in monkeys and humans. Physiology 23, 171-179. doi: 10.1152/physiol. 00004.2008

Gilet, A. L., Mella, N., Studer, J., Grühn, D., and Labouvie-Vief, G. (2013). Assessing dispositional empathy in adults: a french validation of the interpersonal reactivity index (IRI). Can. J. Behav. Sci. 45, 42-48.doi: $10.1037 /$ a0030425

Gobbini, M. I., Gentili, C., Ricciardi, E., Bellucci, C., Salvini, P., Laschi, C., et al. (2011). Distinct neural systems involved in agency and animacy detection. J. Cogn. Neurosci. 23, 1911-1920. doi: 10.1162/jocn.2010. 21574

Goldman, R. I., Stern, J. M., Engel, J. Jr., and Cohen, M. S. (2002). Simultaneous EEG and fMRI of the $\alpha$ rhythm. Neuroreport 13, 2487-2492. doi: 10.1097/01. WNR.0000047685.08940.d0

Gruzelier, J. H. (2014). EEG-neurofeedback for optimising performance. I: a review of cognitive and affective outcome in healthy participants. Neurosci. Biobehav. Rev. 44, 124-141. doi: 10.1016/j.neubiorev.2013.09.015

Han, S., Fan, Y., and Mao, L. (2008). Gender difference in empathy for pain: an electrophysiological investigation. Brain Res. 1196, 85-93. doi: 10.1016/j. brainres.2007.12.062

Hari, R., Forss, N., Avikainen, S., Kirveskari, E., Salenius, S., and Rizzolatti, G. (1998). Activation of human primary motor cortex during action observation: a neuromagnetic study. Proc. Natl. Acad. Sci. U S A 95, 15061-15065. doi: $10.1073 /$ pnas.95.25.15061

Hoenen, M., Lübke, K. T., and Pause, B. M. (2015). Somatosensory mu activity reflects imagined pain intensity of others. Psychophysiology 52, 1551-1558. doi: $10.1111 /$ psyp. 12522

Hoenen, M., Schain, C., and Pause, B. M. (2013). Down-modulation of mu-activity through empathic top-down processes. Soc. Neurosci. 8, 515-524. doi: 10.1080/17470919.2013.833550

Jackson, P. L., Meltzoff, A. N., and Decety, J. (2005). How do we perceive the pain of others? A window into the neural processes involved in empathy. Neuroimage 24, 771-779. doi: 10.1016/j.neuroimage.2004.09.006

Joyal, C. C., Jacob, L., Cigna, M. H., Guay, J. P., and Renaud, P. (2014). Virtual faces expressing emotions: an initial concomitant and construct validity study. Front. Hum. Neurosci. 8:787. doi: 10.3389/fnhum.2014.00787

Keysers, C., and Gazzola, V. (2010). Social neuroscience: mirror neurons recorded in humans. Curr. Biol. 20, R353-R354. doi: 10.1016/j.cub.2010.03.013

Metting Van Rijn, A. C., Kuiper, A. P., Dankers, T. E., and Grimbergen, C. A. (1996). "Low-cost active electrode improves the resolution in biopotential recordings," in Proceedings of the 18th Annual International Conference of the IEEE Engineering in Medicine and Biology Society (Vol. 1) (Amsterdam, Netherlands: IEEE), 101-102.

Moll, J., Weingartner, J. H., Bado, P., Basilio, R., Sato, J. R., Melo, B. R., et al. (2014). Voluntary enhancement of neural signatures of affiliative emotion using FMRI neurofeedback. PLoS One 9:e97343. doi: 10.1371/journal.pone.0097343

Moore, A., Gorodnitsky, I., and Pineda, J. (2012). EEG mu component responses to viewing emotional faces. Behav. Brain Res. 226, 309-316. doi: 10.1016/j.bbr. 2011.07.048

Muthukumaraswamy, S. D., Johnson, B. W., and McNair, N. A. (2004). Mu rhythm modulation during observation of an object-directed grasp. Cogn. Brain Res. 19, 195-201. doi: 10.1016/j.cogbrainres.2003.12.001

Ono, M., Fujita, M., and Yamada, S. (2009). Physiological and psychological responses to expressions of emotion and empathy in post-stress communication. J. Physiol. Anthropol. 28, 29-35. doi: 10.2114/ jpa2.28.29

Paiva, A., Dias, J., Sobral, D., Aylett, R., Woods, S., Hall, L., et al. (2005). Learning by feeling: evoking empathy with synthetic characters. Appl. Artif. Intell. 19, 235-266. doi: 10.1080/08839510590910165

Peng, W., Babiloni, C., Mao, Y., and Hu, Y. (2015). Subjective pain perception mediated by $\alpha$ rhythms. Biol. Psychol. 109, 141-150. doi: 10.1016/j.biopsycho. 2015.05.004

Perry, A., Bentin, S., Bartal, I. B. A., Lamm, C., and Decety, J. (2010). "Feeling” the pain of those who are different from us: modulation of EEG in the mu/ $\alpha$ range. Cogn. Affect. Behav. Neurosci. 10, 493-504. doi: 10.3758/CABN.10.4.493
Perry, A., Stein, L., and Bentin, S. (2011). Motor and attentional mechanisms involved in social interaction-evidence from mu and $\alpha$ EEG suppression. Neuroimage 58, 895-904. doi: 10.1016/j.neuroimage.2011.06.060

Pfurtscheller, G., and Lopes da Silva, F. H. (1999). Event-related EEG/MEG synchronization and desynchronization: basic principles. Clin. Neurophysiol. 110, 1842-1857. doi: 10.1016/s1388-2457(99)00141-8

Pineda, J. A. (2005). The functional significance of mu rhythms: translating "seeing" and "hearing" into "doing". Brain Res. Rev. 50, 57-68. doi: 10.1016/j. brainresrev.2005.04.005

Pineda, J. O. A., and Oberman, L. M. (2006). What goads cigarette smokers to smoke? Neural adaptation and the mirror neuron system. Brain Res. 1121, 128-135. doi: 10.1016/j.brainres.2006.08.128

Preston, S. D., and de Waal, F. B. (2002). Empathy: its ultimate and proximate bases. Behav. Brain Sci. 25, 1-20. doi: 10.1017/s0140525x020 00018

Rizzolatti, G., and Craighero, L. (2004). The mirror-neuron system. Аnnu. Rev. Neurosci. 27, 169-192. doi: 10.1146/annurev.neuro.27.070203. 144230

Schulte-Rüther, M., Markowitsch, H. J., Shah, N. J., Fink, G. R., and Piefke, M. (2008). Gender differences in brain networks supporting empathy. Neuroimage 42, 393-403. doi: 10.1016/j.neuroimage.2008.04.180

Simkin, D. R., Thatcher, R. W., and Lubar, J. (2014). Quantitative EEG and neurofeedback in children and adolescents: anxiety disorders, depressive disorders, comorbid addiction and attention-deficit/hyperactivity disorder, and brain injury. Child Adolesc. Psychiatr. Clin. N. Am. 23, 427-464. doi: 10.1016/j.chc.2014.03.001

Thut, G., Nietzel, A., Brandt, S. A., and Pascual-Leone, A. (2006). $\alpha$-band electroencephalographic activity over occipital cortex indexes visuospatial attention bias and predicts visual target detection. J. Neurosci. 26, 9494-9502. doi: 10.1523/JNEUROSCI.0875-06.2006

Toussaint, L., and Webb, J. R. (2005). Gender differences in the relationship between empathy and forgiveness. J. Soc. Psychol. 145, 673-685. doi: 10.3200/socp.145.6.673-686

Usakli, A. B. (2010). Improvement of EEG signal acquisition: an electrical aspect for state of the art of front end. Computat. Intell. Neurosci. 2010:630649. doi: $10.1155 / 2010 / 630649$

Van de Mortel, T. F. (2008). Faking it: social desirability response bias in self-report research. Aust. J. Adv. Nurs. 25, 40-48.

Vanderwert, R. E., Fox, N. A., and Ferrari, P. F. (2013). The mirror mechanism and mu rhythm in social development. Neurosci. Lett. 540, 15-20. doi: 10.1016/j. neulet.2012.10.006

Whitmarsh, S., Nieuwenhuis, I. L., Barendregt, H. P., and Jensen, O. (2011). Sensorimotor $\alpha$ activity is modulated in response to the observation of pain in others. Front. Hum. Neurosci. 5:91. doi: 10.3389/fnhum.2011. 00091

Woodruff, C. C., Martin, T., and Bilyk, N. (2011). Differences in self- and other-induced $\mathrm{Mu}$ suppression are correlated with empathic abilities. Brain Res. 1405, 69-76. doi: 10.1016/j.brainres.2011.05.046

Yang, C. Y., Decety, J., Lee, S., Chen, C., and Cheng, Y. (2009). Gender differences in the mu rhythm during empathy for pain: an electroencephalographic study. Brain Res. 1251, 176-184. doi: 10.1016/j.brainres.2008. 11.062

Yao, S., Becker, B., Geng, Y., Zhao, Z., Xu, X., Zhao, W., et al. (2016). Voluntary control of anterior insula and its functional connections is feedback-independent and increases pain empathy. Neuroimage 130, 230-240. doi: 10.1016/j.neuroimage.2016.02.035

Conflict of Interest Statement: The authors declare that the research was conducted in the absence of any commercial or financial relationships that could be construed as a potential conflict of interest.

Copyright (c) 2018 Joyal, Neveu, Boukhalfi, Jackson and Renaud. This is an open-access article distributed under the terms of the Creative Commons Attribution License (CC BY). The use, distribution or reproduction in other forums is permitted, provided the original author(s) and the copyright owner(s) are credited and that the original publication in this journal is cited, in accordance with accepted academic practice. No use, distribution or reproduction is permitted which does not comply with these terms. 